\title{
MeV dark matter and small scale structure
}

\author{
Dan Hooper, ${ }^{1}$ Manoj Kaplinghat, ${ }^{2}$ Louis E. Strigari, ${ }^{2}$ and Kathryn M. Zurek ${ }^{3}$ \\ ${ }^{1}$ Theoretical Astrophysics, Fermi National Accelerator Laboratory, Batavia, Illinois 60510, USA \\ ${ }^{2}$ Center for Cosmology, Department of Physics and Astronomy, University of California, Irvine, California 92697, USA \\ ${ }^{3}$ Phenomenology Institute, University of Wisconsin, Madison, Wisconsin 53706, USA
}

(Received 1 May 2007; published 8 November 2007)

\begin{abstract}
Weakly interacting massive particles with electroweak scale masses (neutralinos, etc.) remain in kinetic equilibrium with other particle species until temperatures approximately in the range of $10 \mathrm{MeV}$ to $1 \mathrm{GeV}$, leading to the formation of dark matter substructure with masses as small as $10^{-4} M_{\odot}$ to $10^{-12} M_{\odot}$. However, if dark matter consists of particles with MeV scale masses, as motivated by the observation of $511 \mathrm{keV}$ emission from the galactic bulge, such particles are naturally expected to remain in kinetic equilibrium with the cosmic neutrino background until considerably later times. This would lead to a strong suppression of small scale structure with masses below about $10^{7} M_{\odot}$ to $10^{4} M_{\odot}$. This cutoff scale has important implications for present and future searches for faint local group satellite galaxies and for the missing satellites problem. We also summarize current constraints on the $\mathrm{MeV}$ dark matter scenario.
\end{abstract}

DOI: 10.1103/PhysRevD.76.103515

PACS numbers: 95.35.+d, 98.35.Gi, 98.62.Gq

\section{INTRODUCTION}

In the standard cosmology, featuring cold, collisionless dark matter, structures form hierarchically with the smallest mass objects forming first and progressively larger objects forming via subsequent mergers and accretion. This paradigm has been remarkably successful at describing the observed large scale structure.

Within this paradigm, the mass of the smallest dark matter halos depends on the mass of the dark matter particles, and on the temperature at which they decouple kinetically from other particle species. For a typical weakly interacting massive particle (WIMP) with an electroweak scale mass, kinetic decoupling from the standard model leptons occurs at a temperature in the range of roughly $10 \mathrm{MeV}$ to $1 \mathrm{GeV}$, leading to the formation of structures with masses as small as $10^{-4} M_{\odot}$ to $10^{-12} M_{\odot}[1,2]$. If dark matter consists of particles which remain in kinetic equilibrium with neutrinos until later times (lower temperatures), the smallest dark matter halos will be considerably more massive than are predicted for WIMPs with electroweak scale masses $[3,4]$.

Dark matter particles with $\mathrm{MeV}$ scale masses have been previously motivated by the observation of $511 \mathrm{keV}$ emission from the galactic bulge [5,6]. In particular, annihilating $\mathrm{MeV}$ dark matter can inject the required rate of positrons into the galactic bulge, and also be produced in the early universe with the measured dark matter abundance [6]. Dark matter in the form of $\mathrm{MeV}$ mass scalars, $\phi$, annihilating through the exchange of a light gauge boson, $U$, can accommodate these requirements [6-8]. Constraints on this scenario have been placed by colliders [9,10], neutrino experiments [11], atomic physics experiments [12], observations of supernova 1987A [13], the $511 \mathrm{keV}$ line width [14] and big bang nucleosynthesis (BBN) [15].
In this article, we revisit the $\mathrm{MeV}$ dark matter scenario, and calculate the resulting matter power spectrum. We find that if the $U$ boson's couplings to neutrinos is similar to its couplings to electrons, the matter power spectrum is suppressed on small scales, leading to an absence of dark matter halos with masses below about $10^{7} M_{\odot}$ to $10^{4} M_{\odot}$. We show that this suppression scale is consistent with the region of parameter space where the $\mathrm{MeV}$ dark matter has the correct relic abundance as determined by recent cosmological observations [16].

\section{THE MEV DARK MATTER POWER SPECTRUM}

To calculate the power spectrum for $\mathrm{MeV}$ dark matter, we start by determining the temperature at which kinetic decoupling occurs. The squared amplitude for dark matterneutrino elastic scattering is given by

$$
\left|\mathcal{M}_{\phi \nu}\right|^{2}=\frac{8 g_{U \phi \phi}^{2} g_{U \nu \nu}^{2} m_{\phi}^{2} E_{\nu}^{2}}{\left(t-m_{U}^{2}\right)^{2}}[1+\cos \theta]
$$

where $t=-2 E_{\nu}^{2}(1-\cos \theta), m_{\phi}$ is the mass of the dark matter particle, $m_{U}$ is the mass of the exchanged boson, and $g_{U \phi \phi}$ and $g_{U \nu \nu}$ are that boson's couplings to dark matter and neutrinos, respectively. This leads to an elastic scattering cross section (in the $m_{U} \gg E_{\nu}$ limit) of

$$
\sigma_{\phi \nu}=\frac{g_{U \phi \phi}^{2} g_{U \nu \nu}^{2} E_{\nu}^{2}}{2 \pi m_{U}^{4}}
$$

To determine the temperature of kinetic decoupling for the dark matter particle, we solve the Boltzmann equation, including the $\phi-\nu$ collision term. The resulting equation $[17,18]$ is

$$
\frac{d f(\vec{p})}{d t}=\Gamma\left(T_{\nu}\right)\left(T_{\nu} m_{\phi} \nabla_{\vec{p}}^{2}+\vec{p} \cdot \nabla_{\vec{p}}+3\right) f(\vec{p}),
$$

where $\Gamma=31 \pi^{3} g_{U \phi \phi}^{2} g_{U \nu \nu}^{2} T_{\nu}^{6} /\left(42 m_{U}^{4} m_{\phi}\right)$ is the rate for 
the dark matter distribution function to relax to its equilibrium value. An intuitive approximation to this relaxation rate is $\dot{E}_{k} / E_{k}=(4 \pi)^{-1} \int d E_{\nu} d \Omega\left(d n_{\nu} / d E_{\nu}\right) \times$ $\left(d \sigma_{\phi \nu} / d \Omega\right) \delta E_{k} / E_{k}$ where $d n_{\nu} / d E_{\nu}$ is the differential number density of all neutrinos, $E_{k}=|\vec{p}|^{2} / 2 m_{\phi}$ is the kinetic energy of dark matter particles and $\delta E_{k}$ is the kinetic energy transferred per collision. This approximation yields $15.2 g_{U \phi \phi}^{2} g_{U \nu \nu}^{2} T_{\nu}^{6} /\left(m_{U}^{4} m_{\phi}\right)$ for $E_{k}=3 T_{\nu} / 2$, which is a factor of about 1.5 smaller than the exact result.

Equation (3) is solved by a Boltzmann distribution with a temperature for the dark matter particle that scales as $T_{\nu}$ during the strongly coupled regime and as $T_{\nu}^{2}$ after decoupling. We define the kinetic decoupling temperature as $T_{\mathrm{kd}}=T_{\nu}$, such that $\Gamma\left(T_{\nu}\right)=H\left(T_{\nu}\right) \approx 5.97 \sqrt{G_{N}} T_{\nu}^{2}$. This gives us

$$
T_{\mathrm{kd}}=2.1 \mathrm{keV} \frac{m_{U}}{\mathrm{MeV}}\left(\frac{m_{\phi}}{\mathrm{MeV}}\right)^{1 / 4}\left(\frac{10^{-6}}{g_{U \phi \phi} g_{U \nu \nu}}\right)^{1 / 2} .
$$

Using the above decoupling temperature and a fluid description similar to Ref. [2], we have calculated the power spectrum of $\mathrm{MeV}$ dark matter. Bertschinger [17] has shown that this fluid description provides a good approximation to the solution of the full Fokker-Planck equations if the correct decoupling temperature (as calculated above) is used.

In order to make the numerical solution tractable at early times, we found the tight coupling solution for the slip between the dark matter and neutrino bulk velocities to second order. We switch to the exact fluid equations (analogous to the baryon-photon fluid equations [19]) well before decoupling. For late times (much after decoupling), we use the explicit free-streaming solution $[2,17]$ to evolve the dark matter perturbations to the present.

The interactions, subsequent decoupling and evolution of the dark matter particles damp the matter power spectrum. The coupling of the dark matter to other particle species (here neutrinos) will lead to damped $[3,20]$ oscillatory features [2]. This damping effect dominates for the case of WIMPs with electroweak scale masses. After decoupling, the free-streaming of the dark matter particles further suppresses the power spectrum. For MeV dark matter, this effect dominates for the viable region of parameter space where $T_{\mathrm{kd}} \gtrsim \mathrm{keV}$ and $T_{\mathrm{kd}} / m_{\phi} \gtrsim 10^{-3}$. Also, analogous to the Silk damping effect for photons, as neutrinos kinetically decouple from dark matter they begin to free-stream and damp perturbations. However, the scale associated with this damping is much smaller than the other damping scales since neutrinos decouple from dark matter well before a keV; this effect is subdominant and not included in our calculation.

In Fig. 1, we show the effect on the matter power spectrum of $\mathrm{MeV}$ dark matter as compared to that for the standard cold dark matter case. Large wave numbers are strongly suppressed, resulting in a reduced number of small dark matter halos. Also shown in the figure as a

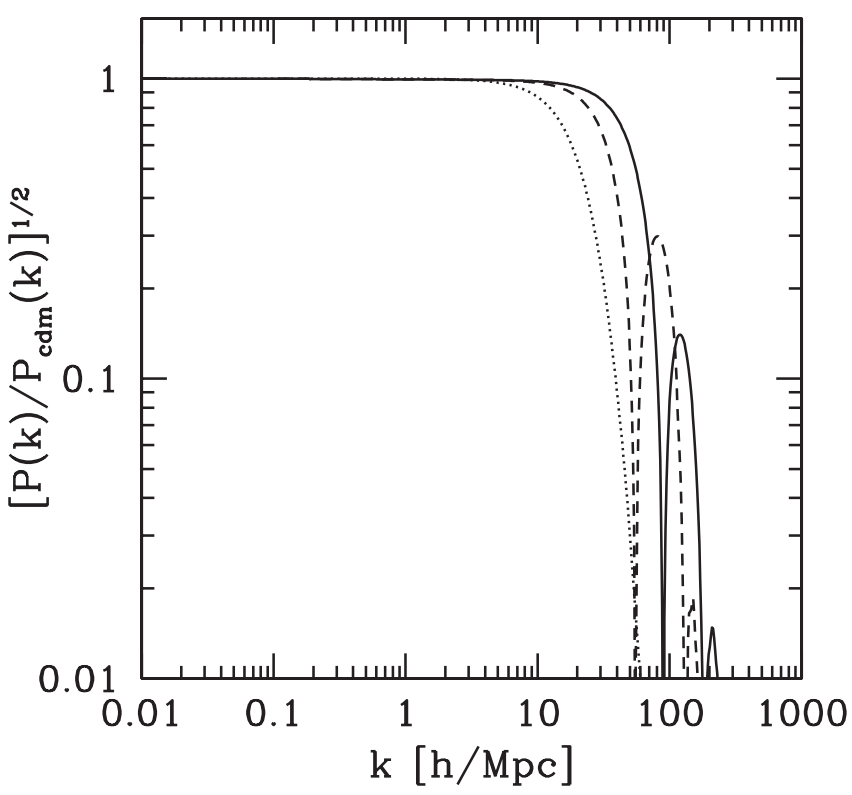

FIG. 1. The effect of $\mathrm{keV}$ scale kinetic decoupling on the matter power spectrum, as predicted in $\mathrm{MeV}$ dark matter models. Shown are results for a $1 \mathrm{MeV}$ dark matter particle with a $10 \mathrm{keV}$ (solid) and $1.0 \mathrm{keV}$ (dashed) kinetic decoupling temperature. The dotted line denotes the limit relevant for warm dark matter, as inferred from lyman-alpha forest observations [21].

dotted curve is the (strictest) limit found for the case of warm dark matter from observations of the lyman-alpha forest [21].

For $T_{\mathrm{kd}} \gtrsim \mathrm{keV}$ and $T_{\mathrm{kd}} / m_{\phi} \gtrsim 10^{-3}$, the scale at which the power spectrum is truncated is closely related to the canonical free-streaming scale (mean comoving distance traveled by a dark matter particle from decoupling to matter-radiation equality),

$$
k_{f}^{-1}=2.5 \mathrm{kpc}\left(\frac{\mathrm{keV}}{T_{\mathrm{kd}}}\right)^{1 / 2}\left(\frac{\mathrm{MeV}}{m_{\phi}}\right)^{1 / 2} \ln \left(4 a_{\mathrm{EQ}} / a_{\mathrm{kd}}\right) \text {, }
$$

where $a_{\mathrm{kd}}$ and $a_{\mathrm{EQ}}$ are the scale factors at decoupling and matter-radiation equality, respectively. The suppression of the dark matter power spectrum on scales smaller than $k_{f}^{-1}$, in turn, leads to a cutoff in the mass function of dark matter halos. Compared to the case with no cutoff, one would find a paucity of halos with masses less than roughly $4 \pi\left(\pi / k_{f}\right)^{3} \rho_{M} / 3$, where $\rho_{M}$ is the present cosmological matter density. To obtain a more accurate estimate, we find the mass at which the expected number of dark matter halos falls by a factor of $e$ compared to the prediction for dark matter particles with electroweak scale masses. We calculate the mass function of dark matter halos using the Press-Schechter prescription. We note that the validity of this prescription for power spectra with sharply truncated power (as found in our scenario) has not been conclusively demonstrated. Nevertheless, the cutoff mass derived here is useful in the sense that it highlights the mass scale below 
which we expect deviations from the predictions of standard cold dark matter. We find this cutoff mass to be

$$
M_{c} \sim 3 \times 10^{7} M_{\odot}\left(\frac{T_{\mathrm{kd}}}{\mathrm{keV}}\right)^{-3 / 2}\left(\frac{m_{\phi}}{\mathrm{MeV}}\right)^{-3 / 2} .
$$

Combining this expression with our specific particle physics scenario, we arrive at the estimate:

$$
M_{c} \sim 10^{7} M_{\odot}\left(\frac{m_{U}}{\mathrm{MeV}}\right)^{-3 / 2}\left(\frac{m_{\phi}}{\mathrm{MeV}}\right)^{-15 / 8}\left(\frac{g_{U \phi \phi} g_{U \nu \nu}}{10^{-6}}\right)^{3 / 4} .
$$

We note that for $T_{\mathrm{kd}} \sim \mathrm{keV}$ and $m_{\phi} \sim \mathrm{MeV}$, the smallest halos that form (those with mass $\sim M_{c}$ ) are the ones that host the smallest of the dwarf galaxies seen in the Milky Way [22]. Therefore, the predictions for the number of satellites in this scenario will be different than that for dark matter with electroweak scale masses. High resolution numerical simulations of truncated power spectra models and a detailed treatment of galaxy formation on small scales will be required to make robust predictions for the satellite (dwarf) galaxy population in galaxies like the Milky Way and Andromeda.

\section{RELIC ABUNDANCE AND OTHER CONSTRAINTS}

There are a number of constraints on the various couplings and masses. First, we require that $\mathrm{MeV}$ dark matter is thermally produced in the early universe with the observed abundance [16]. The annihilation cross section for scalar dark matter particles through the $s$-channel exchange of a $U$-boson is [7,9]:

$$
\begin{aligned}
\sigma v= & \frac{g_{U \phi \phi}^{2}\left(s-4 m_{\phi}^{2}\right)}{12 \pi s\left[\left(s-m_{U}^{2}\right)^{2}+\Gamma_{U}^{2} m_{U}^{2}\right]} \\
& \times \sum_{f} \sqrt{1-4 m_{f}^{2} / s}\left[s\left(g_{f_{L}}^{2}+g_{f_{R}}^{2}\right)\right. \\
& \left.+m_{f}^{2}\left(6 g_{f_{L}} g_{f_{R}}-\left(g_{f_{L}}^{2}+g_{f_{R}}^{2}\right)\right)\right]
\end{aligned}
$$

where we have denoted the $U$ couplings to left and righthanded fermions by $g_{f_{L}}$ and $g_{f_{R}}$, respectively. The sum is over $e^{+} e^{-}$and the three species of neutrinos. Notice that at low velocities $\left(s \approx 4 m_{\phi}^{2}\right)$ the cross section approaches zero, being entirely the result of a P-wave amplitude.

In order for dark matter annihilations to generate the observed $511 \mathrm{keV}$ photons from the galactic bulge, positrons must be injected with energies no greater than $\sim 3 \mathrm{MeV}$ (more energetic positrons would unacceptably broaden the $511 \mathrm{keV}$ line width). This leads to the constraint, $0.511 \mathrm{MeV} \lesssim \mathrm{m}_{\phi} \lesssim 3 \mathrm{MeV}$ [14]. We also require that $m_{\phi}<m_{U}$ in order to avoid dark matter annihilating largely to $U U$, which is not s-wave suppressed [23]. For a 0.511-3 MeV dark matter particle to be generated in a quantity consistent with the observed dark matter abun-

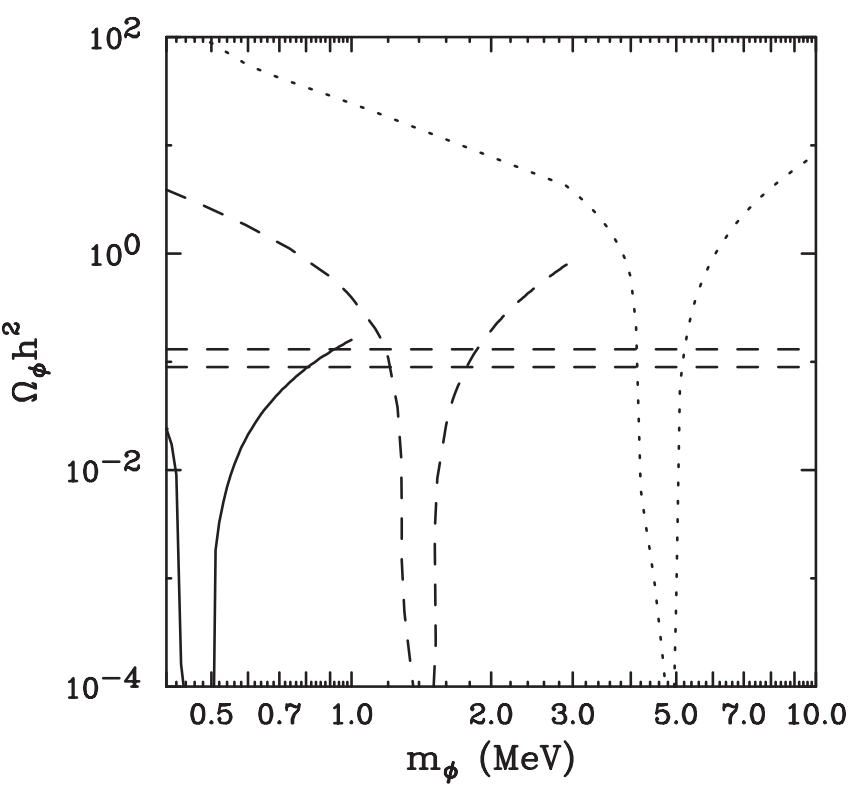

FIG. 2. The thermal relic abundance of dark matter as a function of its mass for $m_{U}=1 \mathrm{MeV}$ (solid), $3 \mathrm{MeV}$ (dashed) and $10 \mathrm{MeV}$ (dotted). In each case, the product $g_{U \phi \phi} g_{U f f}$ was set to $10^{-6}$ for each of $f=e, \nu_{e}, \nu_{\mu}$ and $\nu_{\tau}$. The dashed horizontal lines denote the measured density of dark matter [16]. We show only the results for the viable range, $m_{\phi}<m_{U}$ [23].

dance, an annihilation cross section on the order of a picobarn is required during the freeze-out epoch.

We show in Fig. 2 the abundance of dark matter in this model as a function of its mass, for three values of the gauge boson mass, and for couplings of $g_{U \phi \phi} \times g_{U f f}=$ $10^{-6}$. Throughout, we adopt a common $U$-fermionfermion coupling for electrons and neutrinos.

The $U$-boson's couplings to fermions are constrained by $\nu e$ scattering experiments such that $g_{U \nu \nu} \sqrt{g_{U e_{L} e_{L}}^{2}+g_{U e_{R} e_{R}}^{2}} \lesssim m_{U}^{2} G_{F}[7,8]$. For the case of a common $U$-fermion-fermion coupling, this reduces to $g_{U f f} \lesssim 2.9 \times 10^{-6} \times\left(m_{U} / \mathrm{MeV}\right)^{2}$. A somewhat weaker constraint can be found from measurements of the electron's magnetic moment [7].

In Fig. 3, we show the range of $m_{U}$ and the product $g_{U \phi \phi} \times g_{U f f}$ for which the measured dark matter density can be made to match the thermal relic abundance in this model (for some value of $m_{\phi}$ in the range of $m_{e}$ to $3 \mathrm{MeV}$ ). We also show the constraints from $\nu e$ scattering experiments (for the minimally restrictive case of $g_{U \phi \phi} \approx 1$ ) $[7,8]$ and from the measurement of the electron's magnetic moment [7].

As light blue (gray) lines, we have plotted contours of constant $M_{c}$ from $10^{7}$ to $10^{4}$ solar masses. Here, we have used $m_{\phi}=1 \mathrm{MeV}$. For other masses, the results vary as $M_{c} \propto m_{\phi}^{-15 / 8}$. From this figure, we see that once all of the constraints are considered, $M_{c}$ is generally expected to fall 


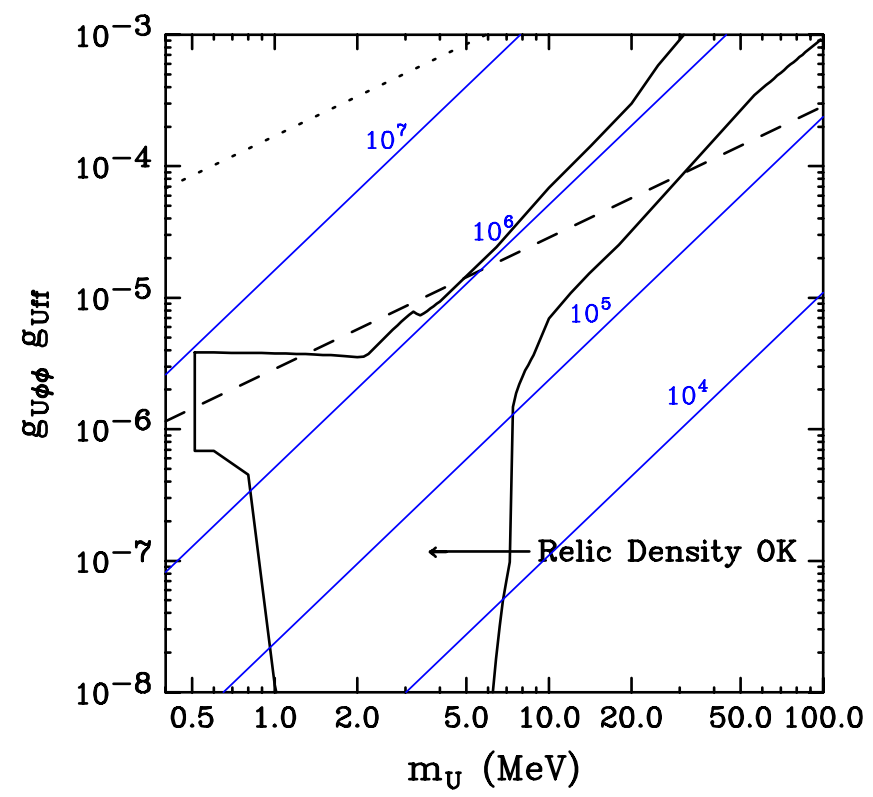

FIG. 3 (color online). Regions in the $m_{U}$ versus $g_{U \phi \phi} g_{U f f}$ plane in which the measured dark matter density matches the thermal relic abundance for some value of $m_{\phi}$ in the range of $m_{e}$ to $3 \mathrm{MeV}$. We have adopted a common $U$-fermion-fermion coupling for electrons and neutrinos. The dashed line denotes the constraint from $\nu e$ scattering experiments (for the minimally restrictive case of $\left.g_{U \phi \phi} \approx 1\right)$ [7,8]. The dotted line denotes the (weaker) constraint from measurements of the electron's magnetic moment [7]. The light blue (gray) lines are contours of constant $M_{c}$ from $10^{4}$ to $10^{7}$ solar masses. Here, we have used $m_{\phi}=1 \mathrm{MeV}$. For other masses, the results vary as $M_{c} \propto$ $m_{\phi}^{-15 / 8}$. From this figure, we see that once all of the constraints are considered, $M_{c}$ in the range of $10^{4} M_{\odot}$ to $10^{7} M_{\odot}$ are generally expected.

in the range of $10^{4}$ to $10^{7} M_{\odot}$. It should be noted that the region where $m_{U} \approx 1-6 \mathrm{MeV}$ and $g_{U \phi \phi} g_{U f f} \lesssim 10^{-7}$ is highly fine tuned and relies on being very close to the resonance at $2 m_{\phi} \simeq m_{U}$ to avoid the overproduction of dark matter.

The coupling of $\mathrm{MeV}$ dark matter to the neutrinos and electrons could change BBN predictions. Effects of this nature have been considered previously but those results [15] are not directly applicable to our model. We have checked that there are viable regions of parameter space where the expected deviations from standard BBN predictions are within observational bounds [24]. In particular, the strongest constraint is derived from the relative deuterium abundance and can be satisfied if the electrons decouple from the dark matter at a temperature around or greater than $\mathcal{O}(100 \mathrm{keV})$, which in turn requires nonuniversal couplings of the fermions to the dark matter.

$\mathrm{MeV}$ dark matter (and associated $U$-boson) with couplings to neutrinos would have other observable consequences. The existence of such a $U$-boson would lead to $\mathrm{TeV}$ scale absorption features in the high-energy cosmic neutrino spectrum [25]. The spectrum of neutrinos produced in type Ia supernovae could also be modified due to their interactions with dark matter particles produced during the collapse [13]. The residual annihilations during the recombination epoch could produce signatures in the cosmic microwave background observable by the Planck satellite experiment [26].

\section{CONCLUSIONS}

In summary, we have calculated the small scale power spectrum of $\mathrm{MeV}$ dark matter model, motivated by the observation of $511 \mathrm{keV}$ emission from the galactic bulge. Assuming similar relevant couplings for neutrinos and electrons, we find that $\mathrm{MeV}$ dark matter particles remain in kinetic equilibrium with the cosmic neutrino background down to temperatures of $1-10 \mathrm{keV}$. This late kinetic decoupling leads to large free-streaming length for $\mathrm{MeV}$ dark matter and highly suppresses the formation of small scale structure. Depending on the parameters, the matter power spectrum could be cutoff below $10^{7} M_{\odot}$ to $10^{4} M_{\odot}$ in this scenario.

This result has a number of particularly interesting astrophysical implications. First, it predicts a cutoff in the mass function of dwarf galaxies at a mass scale much larger than that for WIMPs with electroweak scale masses. It is known that the number of dwarf galaxy-sized dark matter halos in numerical simulations of cold dark matter is considerably larger than the observed populations in the Milky Way and Andromeda galaxies (i.e. the "missing satellites problem") $[27,28]$. This issue may be resolved by astrophysical means [29] or by altering the nature of the dark matter's interactions [30] or mechanism of production [31]. The model we consider falls into this latter category, though detailed numerical simulations will be required to make precise predictions of the satellite population in $\mathrm{MeV}$ dark matter.

Tests of dark matter models with observations of small scale structure are becoming a realistic possibility given the recent discoveries of faint satellite companions to the Milky Way and Andromeda galaxies [32]. Present estimates of the masses of these new satellites fall in a range that includes the cutoff mass scale in $\mathrm{MeV}$ dark matter models that we discussed. Present and future searches for faint satellites, and the characterization of the mass function at these scales, will thus provide important constraints on the $\mathrm{MeV}$ dark matter scenario.

\section{ACKNOWLEDGMENTS}

We thank John Beacom for discussions on this topic. This work has been supported by the U.S. Department of Energy, including Grant No. DE-FG02-95ER40896, and by NASA Grant No. NAG5-10842, and by NSF Grants No. AST-0607746 and No. PHY-0555689. We acknowledge the Aspen center for Physics where this work was initiated. 
[1] X. 1. Chen, M. Kamionkowski, and X. Zhang, Phys. Rev. D 64, 021302 (2001); V. Berezinsky, V. Dokuchaev, and Y. Eroshenko, Phys. Rev. D 68, 103003 (2003); A. M. Green, S. Hofmann, and D. J. Schwarz, Mon. Not. R. Astron. Soc. 353, L23 (2004); J. Cosmol. Astropart. Phys. 08 (2005) 003; S. Profumo, K. Sigurdson, and M. Kamionkowski, Phys. Rev. Lett. 97, 031301 (2006).

[2] A. Loeb and M. Zaldarriaga, Phys. Rev. D 71, 103520 (2005).

[3] C. Boehm, Int. J. Mod. Phys. A 19, 4355 (2004).

[4] G. Mangano et al., Phys. Rev. D 74, 043517 (2006); C. Boehm et al., arXiv:astro-ph/0309652.

[5] P. Jean et al., Astron. Astrophys. 407, L55 (2003).

[6] C. Boehm, D. Hooper, J. Silk, M. Casse, and J. Paul, Phys. Rev. Lett. 92, 101301 (2004).

[7] C. Boehm and P. Fayet, Nucl. Phys. B683, 219 (2004).

[8] P. Fayet, Phys. Rev. D 70, 023514 (2004).

[9] N. Borodatchenkova, D. Choudhury, and M. Drees, Phys. Rev. Lett. 96, 141802 (2006).

[10] B. McElrath, Phys. Rev. D 72, 103508 (2005); P. Fayet, arXiv:hep-ph/0607094; M. Ablikim et al. (BES Collaboration), Phys. Rev. Lett. 97, 202002 (2006); P. Fayet, Phys. Rev. D 74, 054034 (2006).

[11] C. Boehm, Phys. Rev. D 70, 055007 (2004).

[12] C. Bouchiat and P. Fayet, Phys. Lett. B 608, 87 (2005).

[13] P. Fayet, D. Hooper, and G. Sigl, Phys. Rev. Lett. 96, 211302 (2006).

[14] J. F. Beacom and H. Yuksel, Phys. Rev. Lett. 97, 071102 (2006); J. F. Beacom, N.F. Bell, and G. Bertone, Phys. Rev. Lett. 94, 171301 (2005).

[15] E. W. Kolb, M. S. Turner, and T. P. Walker, Phys. Rev. D 34, 2197 (1986); P. D. Serpico and G. G. Raffelt, Phys. Rev. D 70, 043526 (2004).

[16] D. N. Spergel et al. (WMAP Collaboration), Astrophys. J. Suppl. Ser. 170, 377 (2007).

[17] E. Bertschinger, Phys. Rev. D 74, 063509 (2006).
[18] T. Bringmann and S. Hofmann, J. Cosmol. Astropart. Phys. 07 (2007) 016.

[19] C. P. Ma and E. Bertschinger, Astrophys. J. 455, 7 (1995).

[20] S. Hofmann, D. J. Schwarz, and H. Stoecker, Phys. Rev. D 64, 083507 (2001).

[21] U. Seljak et al., Phys. Rev. Lett. 97, 191303 (2006); M. Viel et al., Phys. Rev. Lett. 97, 071301 (2006).

[22] L.E. Strigari, S.M. Koushiappas, J.S. Bullock, and M. Kaplinghat, Phys. Rev. D 75, 083526 (2007).

[23] C. Jacoby and S. Nussinov, J. High Energy Phys. 05 (2007) 017

[24] R. H. Cyburt, B. D. Fields, K. A. Olive, and E. Skillman, Astropart. Phys. 23, 313 (2005).

[25] D. Hooper, Phys. Rev. D 75, 123001 (2007); S. PalomaresRuiz and T. Weiler (unpublished).

[26] N. Padmanabhan and D. P. Finkbeiner, Phys. Rev. D 72, 023508 (2005).

[27] A. A. Klypin et al., Astrophys. J. 522, 82 (1999).

[28] B. Moore et al., Astrophys. J. 524, L19 (1999).

[29] J.S. Bullock, A. V. Kravtsov, and D. H. Weinberg, Astrophys. J. 539, 517 (2000); A. V. Kravtsov, O. Y. Gnedin, and A. A. Klypin, Astrophys. J. 609, 482 (2004); B. Moore et al., Mon. Not. R. Astron. Soc. 368 , 563 (2006).

[30] C. Boehm, P. Fayet, and R. Schaeffer, Phys. Lett. B 518, 8 (2001); X. l. Chen, S. Hannestad, and R. J. Scherrer, Phys. Rev. D 65, 123515 (2002).

[31] K. Sigurdson and M. Kamionkowski, Phys. Rev. Lett. 92, 171302 (2004); J. A. R. Cembranos et al., Phys. Rev. Lett. 95, 181301 (2005); M. Kaplinghat, Phys. Rev. D 72, 063510 (2005); O. Seto and M. Yamaguchi, Phys. Rev. D 75, 123506 (2007).

[32] B. Willman et al., Astrophys. J. 626, L85 (2005); D. B. Zucker et al. (SDSS Collaboration), Astrophys. J. 643, L103 (2006); V. Belokurov et al. (SDSS Collaboration), Astrophys. J. 654, 897 (2007). 